\title{
Variable Expression of the Stagonospora nodorum Effector SnToxA Among Isolates Is Correlated with Levels of Disease in Wheat
}

\author{
Justin D. Faris, ${ }^{1}$ Zengcui Zhang, ${ }^{2}$ Jack B. Rasmussen, ${ }^{2}$ and Timothy L. Friesen ${ }^{1,2}$ \\ ${ }^{1}$ United States Department of Agriculture-Agricultural Research Service Cereal Crops Unit, 1307 18th Street North, Fargo, \\ ND 58102-2765, U.S.A.; ${ }^{2}$ Department of Plant Pathology, Walster Hall, North Dakota State University, Fargo 58102, U.S.A.
}

Submitted 13 April 2011. Accepted 7 July 2011.

\begin{abstract}
Most research on host-pathogen interactions is focused on mechanisms of resistance, but less is known regarding mechanisms of susceptibility. The wheat-Stagonospora nodorum pathosystem involves pathogen-produced effectors, also known as host-selective toxins, that interact with corresponding dominant host genes to cause disease. Recognition of the $S$. nodorum effectors SnToxA and SnTox 2 is mediated by the wheat genes $T s n 1$ and $S n n 2$, respectively. Here, we inoculated a population of wheat recombinant inbred lines that segregates for Tsn1 and Snn2 with conidia from two $S$. nodorum isolates, $\mathrm{Sn} 4$ and $\mathrm{Sn5}$, which both produce SnToxA and SnTox2 to compare the effects of compatible Tsn1SnToxA and Snn2-SnTox 2 interactions between the two isolates. Genetic analysis revealed that the two interactions contribute equally to disease caused by isolate $\mathrm{Sn} 4$ but the Tsn1SnToxA interaction contributed substantially more to disease conferred by Sn5 than did the Snn2-SnTox2 interaction. Sequence analysis of the SnToxA locus from $\mathrm{Sn} 4$ and Sn5 indicated that they were $99.5 \%$ identical, with no polymorphisms in the coding region or the predicted promoters. Analysis of transcription levels showed that expression levels of SnToxA peaked at $26 \mathrm{~h}$ postinoculation for both isolates but SnToxA expression in Sn5 was more than twice that of Sn4. This work demonstrates that necrotrophic effectors of different isolates can be expressed at different levels in planta, and that higher levels of expression lead to increased levels of disease in the wheat-S. nodorum pathosystem.
\end{abstract}

Stagonospora nodorum blotch (SNB) is caused by the necrotrophic fungus Stagonospora nodorum (Berk.) E. Castell. \& Germano (anamorph: Phaeosphaeria nodorum (E. Müll.) Hedjar). SNB is a major foliar and glume disease of common wheat (Triticum aestivum L., $2 \mathrm{n}=6 \mathrm{x}=42$, AABBDD genomes) and durum wheat (T. turgidum L., $2 \mathrm{n}=4 \mathrm{x}=28$, AABB genomes)

Corresponding author: J. D. Faris; E-mail: justin.faris@ars.usda.gov

GenBank accession numbers HM191251, HM191250, and HM191252 have been assigned to the SnToxA genomic sequences obtained from Stagonospora nodorum isolates Sn4, Sn5, and Sn6, respectively.

Mention of trade names or commercial products in this publication is solely for the purpose of providing specific information and does not imply recommendation or endorsement by the United States Department of Agriculture (USDA). USDA is an equal opportunity provider and employer.

This article is in the public domain and not copyrightable. It may be freely reprinted with customary crediting of the source. The American Phytopathological Society, 2011. in many wheat-growing areas of the world. Because the fungus can infect both the leaves and the glumes, it has the ability to cause significant yield losses (Eyal 1981; King et al. 1983) and negatively impact grain quality (Eyal et al. 1987).

Host-selective toxins (HST) behave as effectors and are important disease determinants in a number of host-pathogen systems involving necrotrophic fungal pathogens (Wolpert et al. 2002). Compatible host-effector interactions usually rely on the recognition of the effector by a dominant host gene, which leads to disease susceptibility and may be referred to as effector-triggered susceptibility (ETS). Absence of either the effector or the dominant host gene precludes recognition and results in an incompatible, or resistant, response. Therefore, these host-effector interactions are the inverse of classical gene-for-gene interactions, where an avirulence effector produced by the pathogen is recognized by a dominant host resistance gene, resulting in a resistance response known as effector-triggered immunity (ETI).

To date, five host-effector interactions have been reported in the wheat-S. nodorum system (Friesen and Faris 2010; Friesen et al. 2008a). These interactions involve the effectors SnToxA, SnTox1, SnTox2, SnTox3, and SnTox4 along with the corresponding host compatibility genes $T s n 1, \operatorname{Snn} 1, \operatorname{Snn} 2, \operatorname{Snn} 3$, and Snn4, respectively. Each of these host gene-effector interactions has been shown to play an important role in conferring disease susceptibility, explaining from $18 \%$ of the variation for the Snn3-SnTox3 interaction (Friesen et al. 2008b) to as much as 95\% of the variation for the Tsn1-SnToxA interaction (Faris and Friesen 2009). In most cases, these interactions have large additive effects when multiple compatible interactions segregate in the same population (Chu et al. 2010; Friesen et al. 2007, 2008b) (e.g,. compatible Tsn1-SnToxA and Snn1-SnTox1 interactions individually explained 28 and $22 \%$, respectively, of the variation in SNB but, together, they explained 48\%) (Chu et al. 2010). However, epistasis has also been observed. Friesen and associates (2008b) showed that compatible Tsn1-SnToxA and Snn2-SnTox2 interactions were epistatic to compatible Snn3SnTox 3 interactions. Thus, the segregation of multiple compatible host-effector interactions in a given population results in the appearance of resistance being quantitatively inherited.

Of the effectors and host genes involved in the five interactions described to date, only the $S$. nodorum effectors SnToxA and SnTox 3 and the wheat gene Tsn 1 have been characterized at the molecular level (Faris et al. 2010; Friesen et al. 2006; Liu et al. 2009), and the Tsn1-SnToxA interaction is the most well characterized. The ToxA gene was likely involved in a horizontal transfer from $S$. nodorum to Pyrenophora tritici-repentis, the necrotrophic fungus that causes the disease tan spot on wheat 
(Friesen et al. 2006). ToxA was first characterized in P. triticirepentis and shown to be a $13-\mathrm{kDa}$ polypeptide (Ballance et al. 1989). Liu and associates (2006) demonstrated that ToxA produced by both pathogens is functionally identical. The wheat Tsnl gene confers sensitivity to ToxA and is located on the long arm of chromosome 5B (Faris et al. 1996, 2000; Haen et al. 2004; Lu et al. 2006). The recent cloning of Tsn1 showed that it has a unique disease resistance gene-like structure in that it harbors serine/threonine protein kinase, nucleotide-binding site (NBS), and leucine-rich repeat (LRR) domains, all in the same transcript (Faris et al. 2010). This and other recent research involving the cloning of effector sensitivity genes in Arabidopsis (Lorang et al. 2007) and sorghum (Nagy and Bennetzen 2008), and research indicating that host response mechanisms in necrotroph-induced ETS and biotroph-induced ETI have much in common (Adhikari et al. 2009; Hammond-Kosack and Rudd 2008; Pandelova et al. 2009), suggests that necrotrophic pathogens may thrive by subverting the resistance mechanisms that plants use to combat pathogens with biotrophic lifestyles.

Here, we conducted research to compare the effects of compatible Tsn1-SnToxA and Snn2-SnTox2 interactions between two different $S$. nodorum isolates on the development of SNB in wheat. We demonstrate that the importance of compatible host gene-effector interactions in conferring susceptibility is variable and isolate dependent. We also show that the magnitude of disease susceptibility attributed to the Tsn1-SnToxA interaction is not due to variation at the sequence level but is correlated with the level of SnToxA gene expression in planta.

\section{RESULTS}

\section{Identification of effectors produced} by $S$. nodorum isolates $\mathrm{Sn} 4$ and $\mathrm{Sn} 5$.

The population of wheat recombinant inbred (RI) lines derived from the hard red spring wheat 'BR34' $\times$ 'Grandin' (BG population) is known to segregate for the genes Tsn1, Snn2, and Snn3, which confer sensitivity to the $S$. nodorum effectors SnToxA, SnTox2, and SnTox3, respectively (Friesen et al. 2008b). Reactions of the BG RI lines to SnToxA and SnTox2 were previously obtained and used to identify individual RI lines with sensitivity to only SnToxA and only SnTox 2 for use as differentials (Friesen et al. 2007). To determine whether the $S$. nodorum isolates $\mathrm{Sn} 4$ and $\mathrm{Sn} 5$ produce SnToxA and SnTox2, culture filtrates were infiltrated into leaves of BR34, Grandin, and the differential lines BG261 (sensitivity to only SnToxA) and BG223 (sensitivity to only SnTox2). The culture filtrates of both $\mathrm{Sn} 4$ and $\mathrm{Sn} 5$ caused necrosis on Grandin, BG261, and BG223 but not on BR34, strongly suggesting that both isolates produced SnToxA and SnTox2 (Fig. 1).

\section{Genetic analysis of Tsn1-SnToxA and}

Snn2-SnTox2 interactions in Sn4 inoculations.

The entire BG population was evaluated for reaction to SNB caused by isolates Sn4 and Sn5 using a completely randomized design with three replicates for each isolate. Disease reaction types were rated using the 0 -to- 5 scale established by Liu and associates (2004b), where $0=$ highly resistant and $5=$ highly susceptible. Using the combined means, the average reaction types of BR34 and Grandin when inoculated with Sn4 were 0.3 and 4.7, respectively, indicating that BR34 was highly resistant to $\mathrm{Sn} 4$ and Grandin was highly susceptible (Table 1). SNB reactions among the RI population ranged from 1.0 to 4.5 , with an average reaction type of 2.8 .

Mean disease reaction types were regressed on the genotypic data in the BG population using quantitative trait loci (QTL) analysis (composite interval mapping) to determine the amount of variation in disease explained by compatible $T_{s n 1}$ SnToxA and Snn2-SnTox 2 interactions. Both interactions were highly significant, with $\log$ of the likelihood (LOD) values greater than 10.0 (Fig. 2; Table 2). This analysis also revealed that the Tsn1-SnToxA interaction explained $25 \%$ of the phenotypic variation and the Snn2-SnTox 2 interaction explained $26 \%$, which indicates that the two interactions contribute equally to disease. Together, the two interactions explained $50 \%$ of the phenotypic variation, indicating that their effects are almost completely additive (Table 3 ).

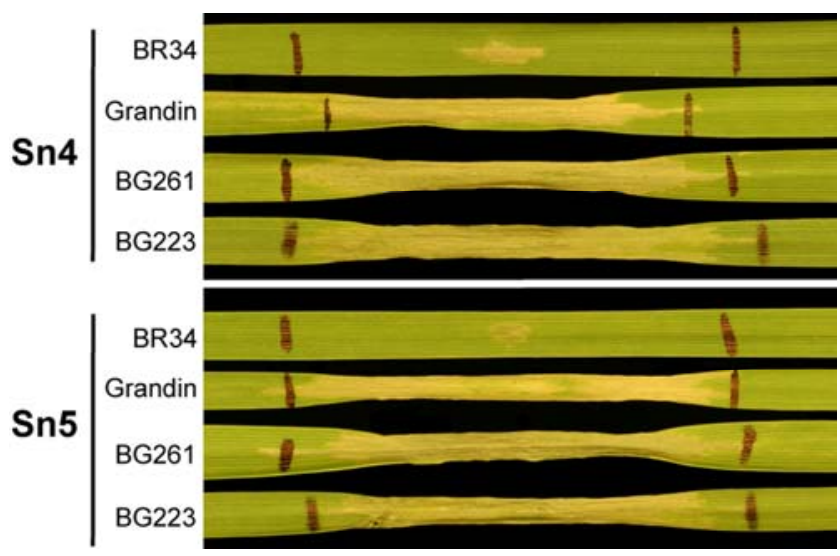

Fig. 1. Leaves of 'BR34', 'Grandin', BG261 (SnToxA differential), and BG223 (SnTox2 differential) infiltrated with culture filtrates of Stagonospora nodorum isolates Sn4 (top panel) and Sn5 (bottom panel).

Table 2. Statistical effects of Tsn1-SnToxA and Snn2-SnTox2 interactions in conferring Stagonospora nodorum blotch caused by Stagonospora nodorum isolates $\mathrm{Sn} 4$ and $\mathrm{Sn} 5$ as determined by quantitative trait loci analysis (composite interval regression mapping)

\begin{tabular}{lccc}
\hline Isolate & Interaction & LOD $^{\mathbf{z}}$ & $\boldsymbol{R}^{\mathbf{2}}$ \\
\hline Sn4 & Tsn1-SnToxA & 10.5 & 0.25 \\
& Snn2-SnTox2 & 10.7 & 0.26 \\
\multirow{2}{*}{ Sn5 } & Tsn1-SnToxA & 20.7 & 0.56 \\
& Snn2-SnTox2 & 3.5 & 0.06 \\
\hline
\end{tabular}

${ }^{\mathrm{z}}$ Experiment-wise log of the likelihood ratio (LOD) significance threshold at the 0.05 level of probability is 3.1 .

Table 1. Average and range of disease reaction types of parents and recombinant inbred (RI) lines of the 'BR34' $\times$ 'Grandin' population for the four allelic state combinations for Tsn1 and Snn2 after inoculation with conidia of Stagonospora nodorum isolates Sn4 and Sn5 ${ }^{\mathrm{Z}}$

\begin{tabular}{|c|c|c|c|c|c|}
\hline \multirow[b]{2}{*}{ Genotype } & \multirow[b]{2}{*}{ Number of RI lines } & \multicolumn{2}{|c|}{ Sn4 } & \multicolumn{2}{|c|}{ Sn5 } \\
\hline & & Reaction type range & Average reaction type & Reaction type range & Average reaction type \\
\hline BR34 & $\cdots$ & $0-1.0$ & $0.3 \pm 0.58$ & $0.5-1.5$ & $1.2 \pm 0.58$ \\
\hline Grandin & $\ldots$ & $4.5-5.0$ & $4.7 \pm 0.29$ & $4.5-5.0$ & $4.8 \pm 0.29$ \\
\hline Tsn1/Snn2 & 30 & $2.7-4.5$ & $3.7 \mathrm{c}$ & $2.7-4.8$ & $3.8 \mathrm{c}$ \\
\hline Tsn1/snn2 & 29 & $1.2-4.3$ & $2.8 \mathrm{~b}$ & $0.8-4.7$ & $3.1 \mathrm{~b}$ \\
\hline tsn1/Snn2 & 28 & $1.7-4.0$ & $2.8 \mathrm{~b}$ & $1.2-2.8$ & $2.0 \mathrm{a}$ \\
\hline tsn1/snn2 & 31 & $1.0-3.2$ & $2.0 \mathrm{a}$ & $0.8-3.5$ & $1.9 \mathrm{a}$ \\
\hline
\end{tabular}

${ }^{\mathrm{z}}$ Numbers followed by the same letter in the same column are not significantly different at the 0.05 level of probability. 
To further define the effects of Tsn1-SnToxA and Snn2SnTox2 interactions, RI lines were separated into different genotypic classes based on $T s n 1$ and $S n n 2$, and average disease reaction types were calculated for each class. Individual RI lines that were sensitive ( $T_{s n} 1$ genotype) and insensitive ( $t s n 1$ genotype) to SnToxA had average reaction types of 3.3 and 2.4 , respectively, and lines sensitive ( $\mathrm{Snn} 2$ genotype) and insensitive (snn2 genotype) to SnTox 2 had average reaction types nearly identical to those for SnToxA (data not shown), which indicates that both interactions contribute equally to disease. Lines insensitive to both SnToxA and SnTox2 (tsnl/snn2 genotype) were significantly more resistant than lines with sensitivity to only one effector (Tsn1/snn2 or $t s n 1 / S n n 2$ genotypes), and lines with sensitivity to both effectors (Tsn1/Snn2 genotype) were significantly more susceptible (Table 1). However, lines with sensitivity to only SnToxA (Tsn1/snn2 genotype) had average reaction types that were the same as those that were sensitive to only SnTox2 (tsn1/Snn2), which again indicates that compatible Tsn1-SnToxA and Snn2-SnTox2 interactions contribute equally to disease caused by $\mathrm{Sn} 4$.

Table 3. Multiple regression model of markers Tsn1 and Snn2 for susceptibility to Stagonospora nodorum blotch caused by Stagonospora nodorum isolate $\mathrm{Sn} 4^{\mathrm{z}}$

\begin{tabular}{lrccc}
\hline Source of variation & $\boldsymbol{d} \boldsymbol{f}$ & $\begin{array}{c}\text { Mean } \\
\text { square }\end{array}$ & $\boldsymbol{F}$ value & $\boldsymbol{P}$ value \\
\hline Tsn1 & 1 & 21.2 & 54.5 & $<0.0001$ \\
Snn2 & 1 & 21.9 & 56.3 & $<0.0001$ \\
Error & 115 & 0.4 & $\ldots$ & $\ldots$ \\
Total & 117 & $\ldots$ & $\ldots$ & $\ldots$ \\
\hline
\end{tabular}

${ }^{\mathrm{z}} R^{2}=0.50$.
Genetic analysis of Tsn1-SnToxA and Snn2-SnTox2 interactions in Sn5 inoculations.

For isolate $\mathrm{Sn} 5$, the average reaction types of BR34 and Grandin were 1.2 and 4.8, respectively, indicating that, as with Sn4, BR34 was highly resistant to Sn5 and Grandin was highly susceptible (Table 1). Reaction types among the population ranged from 0.8 to 4.8 , with an average of 2.7. The QTL analysis indicated that the Snn2-SnTox2 interaction was just above the LOD significance threshold of 3.0, having a value of 3.5 and explaining $6 \%$ of the variation in disease (Fig. 2; Table 2). In contrast, the Tsn1-SnToxA interaction was highly significant, with an LOD value of 20.7 and accounting for $56 \%$ of the phenotypic variation. Therefore, the Tsn1SnToxA interaction was much more significant in the development of SNB caused by Sn5 than was the Snn2-SnTox2 interaction. However, the two interactions together accounted for $60 \%$ of the variation (Table 4 ) indicating, as observed for the Sn4 inoculations, that the effects of compatible Tsn1SnToxA and Snn2-SnTox2 interactions were additive for the Sn5 data.

Table 4. Multiple regression model of markers Tsn1 and Snn2 for susceptibility to Stagonospora nodorum blotch caused by Stagonospora nodorum isolate $\mathrm{Sn} 5^{\mathrm{z}}$

\begin{tabular}{lrccc}
\hline Source of variation & $\boldsymbol{d} \boldsymbol{f}$ & $\begin{array}{c}\text { Mean } \\
\text { square }\end{array}$ & $\boldsymbol{F}$ value & $\boldsymbol{P}$ value \\
\hline Tsn1 & 1 & 66.3 & 154.5 & $<0.0001$ \\
Snn 2 & 1 & 6.3 & 14.8 & 0.0002 \\
Error & 115 & 0.4 & $\ldots$ & $\ldots$ \\
Total & 117 & $\ldots$ & $\ldots$ & $\ldots$ \\
\hline
\end{tabular}

${ }^{\mathrm{z}} R^{2}=0.60$.
Chromosome 2D

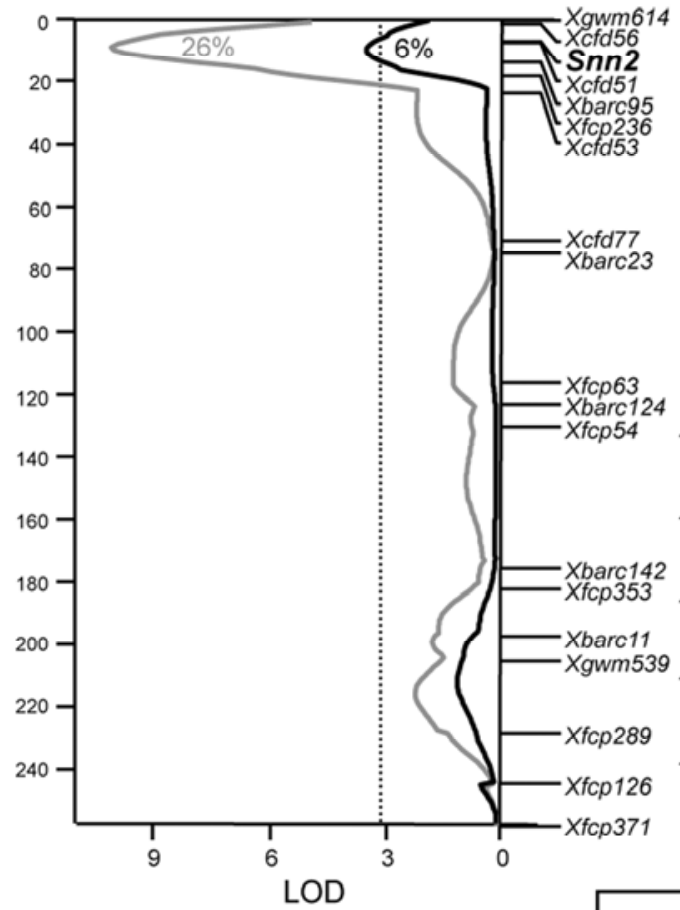

Chromosome 5B

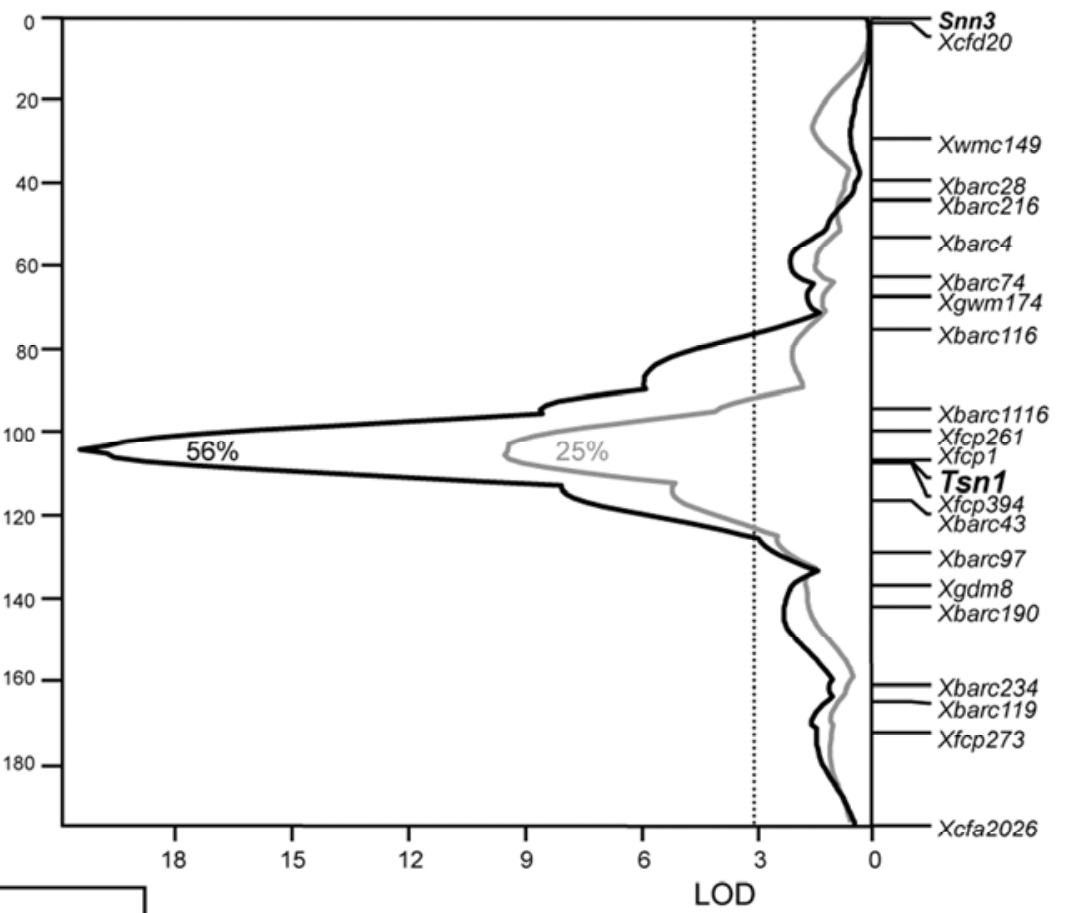

Sn4

Sn5

Fig. 2. Composite interval regression analysis of chromosomes $2 \mathrm{D}$ and $5 \mathrm{~B}$ in the 'BR34' $\times$ 'Grandin' population for reaction to Stagonospora nodorum blotch caused by Stagonospora nodorum isolates Sn4 and Sn5. A centimorgan (cM) scale is indicated to the left of the maps, and the markers are shown in their relative positions along the right. A log of the likelihood ratio (LOD) scale is indicated along the $\mathrm{x}$ axis, and the significant LOD threshold of 3.1 is indicated with a dotted line. Percent variation in disease explained by compatible Tsn1-SnToxA and Snn2-SnTox 2 interactions is indicated near the peaks of the regression lines. 
Sn4-SNTOXA

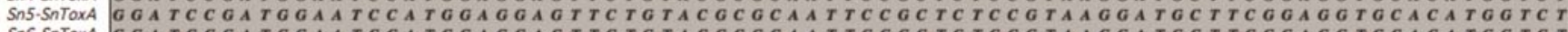
Sn6-SHTOXA GGATCCGATGGAATCCATGGAGGAGTTCTGTACGCGCAATTCCGCTCTCCGTAAGGAGCTCGGAGGTGCACATGGTCT

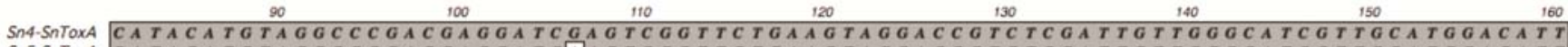

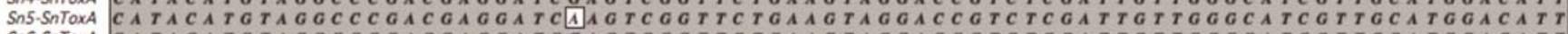
Sn6-SnTOAA

SnA-SnTOXA

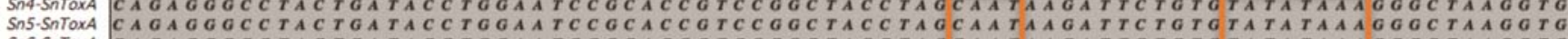

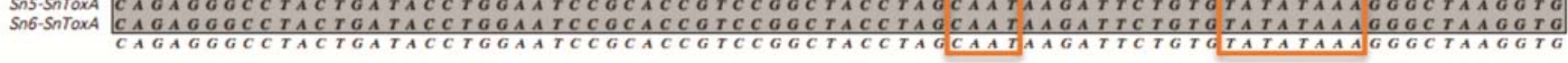
250
SNA-SnTOXA

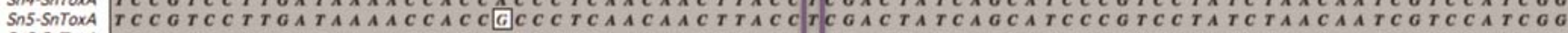

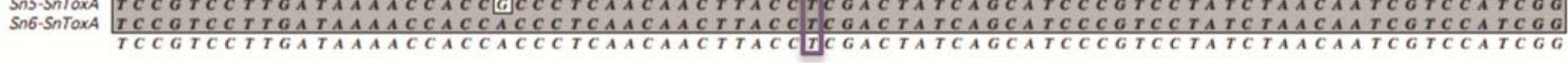

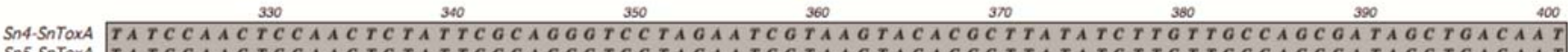

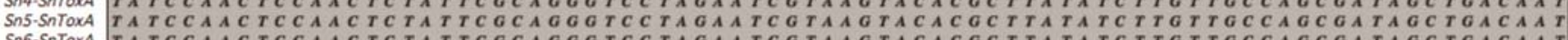
Sn6-SATOXA TATCCAACTCCAACTCTATTCGCAGGGTCCTAGAATCGTAAGTACACGCTTATATCTTGTTGCCAGCGATAGCTGACAAT

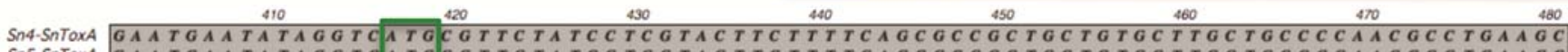

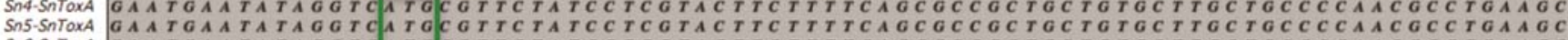

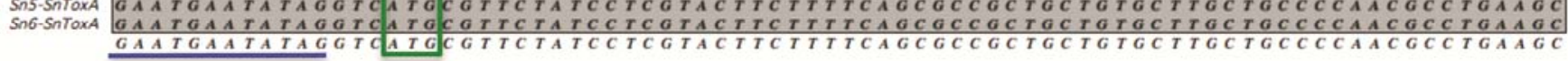

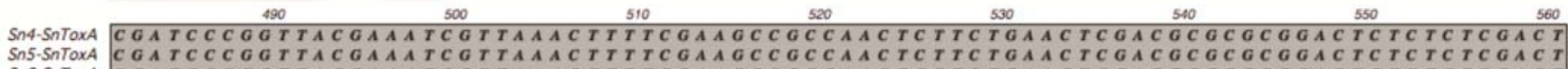

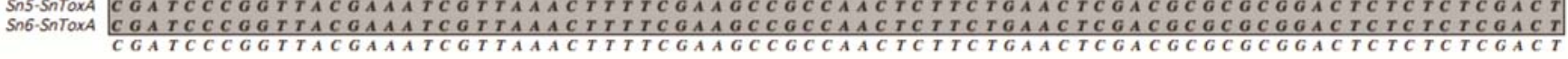

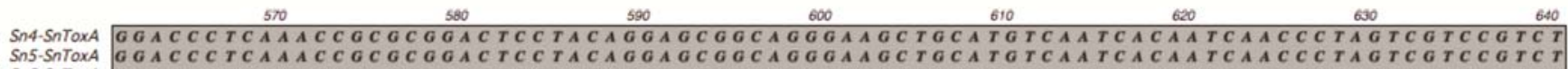

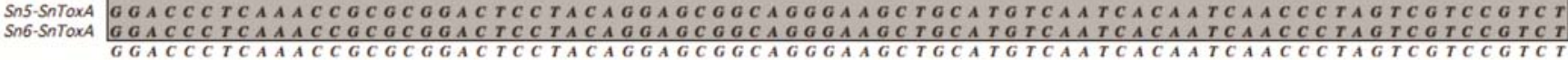
Sn4-SnTOXA

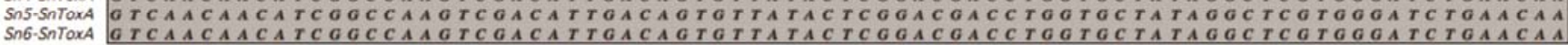
Sn6-SnTOXA GTCAACAACATCGGCCAAGTCGACATTGACAGTGTTATACTCGGACGACCTGGTGCTATAGGCTCGTGGGATCTGAACAA

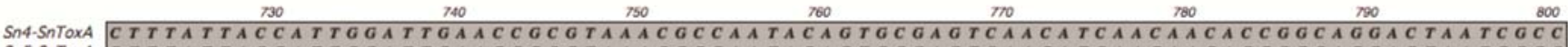

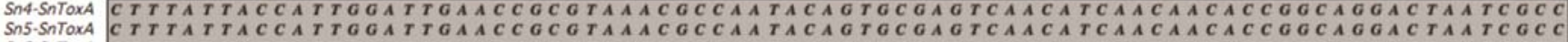
SNG-SATOXA CTRTATTACCATTGGATTGAACCGCGTAAACGCCAATACAGTGCGAGTCAACATCAACAACACCGGCAGGACTAATCGCC

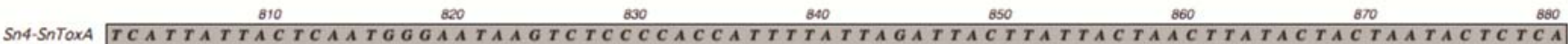

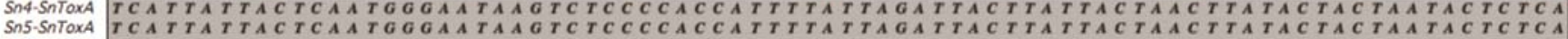

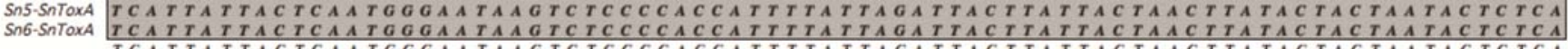
TCATTATTACTCAATGGGAATAAGTCTCCCCACCATTTTATTAGATTACTTATTACTAACTTATACTACTAATACTCTCA Sn4-SinTOXA $990 \quad 900 \quad 910 \quad 920960$

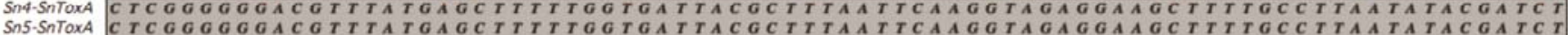

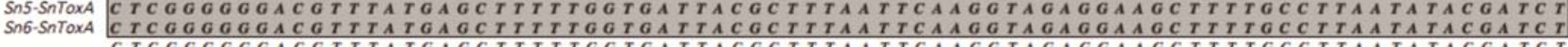
CTCGGGGGGACGTTIATGAGCTTTTTGGTGATTACGCTTTAATTCAAGGTAGAGGAAGCTTTIGCCTTAATATACGATCT

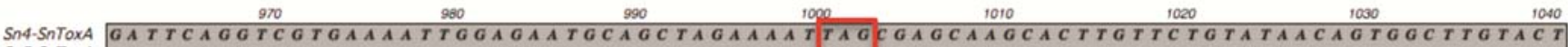

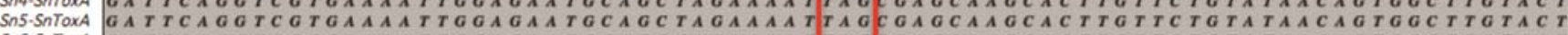
Sn6-SnTOXA GATTCAGGTCGTGAAAATTGGAGAATGCAGCTAGAAAATTAGCGAGCAAGCACTTGTTCTGTATAACAGTGGCTTGTACT GATTCAGGTCGTGAAAATTGGAGAATGCAGCTAGAAAATTAGCGAGCAAGCACTTGTTCTGTATAACAGTGGCTTGTACT

$$
1050 \quad 1060 \quad 1070 \quad 1000 \quad 1090 \quad 1100
$$

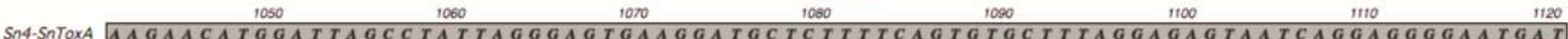

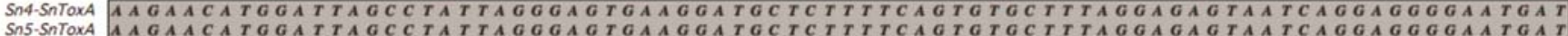

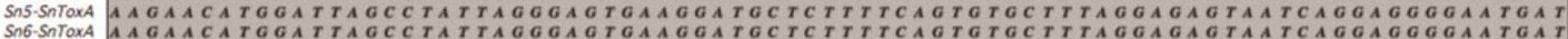
Sn6-SnTOXA AAGAACATGGATTAGCCTATTAGGGAGTGAAGGATGCTCTTTTCAGTGTGCTTRAGGAGAGTAATCAGGAGGGGAATGAT

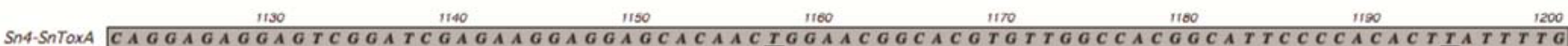

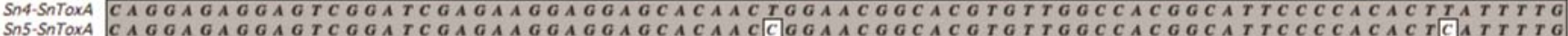

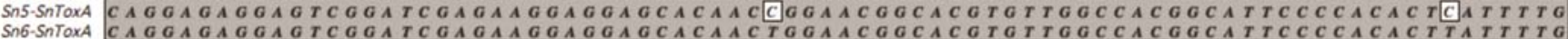
Sn6-SnTOXA CAGGAGAGGAGTCGGATCGAGAAGGAGGAGCACAACTGGAACGGCACGTGTTGGCCACGGCATTCCCCACACTTATTTTG

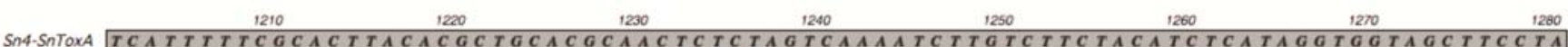

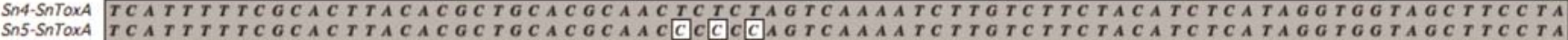

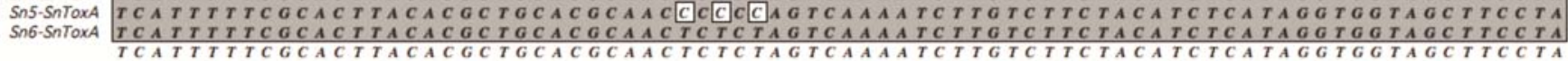

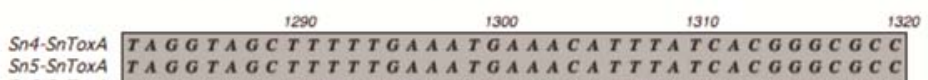

Sn6-SiTOXA $\frac{\text { TAGGTAGCTTTTTGAAATGAAACATTTATCACGGGCGCC }}{\text { TAGGTAGCTTTTTGAAATGAAACATTTATCACGGGCGCC }}$

Fig. 3. DNA sequence alignments of the SnToxA coding and flanking regions from Stagonospora nodorum isolates Sn4, Sn5, and Sn6. Sequences are annotated as described by Ciuffetti and associates (1997). Orange boxes represent putative promoter sequences, the purple box indicates the transcription start site, and the green and red boxes represent the translation start and stop codons, respectively. The two SnToxA introns are underlined in blue. The consensus SnToxA sequence is shown immediately below the aligned sequences.

1422 / Molecular Plant-Microbe Interactions 
Individual RI lines that were sensitive to SnToxA (Tsn I genotype) had average disease reactions of 3.5 caused by $\mathrm{Sn} 5$, whereas lines insensitive to SnToxA ( $t s n 1$ genotype) averaged 2.0. Lines sensitive (Snn2 genotype) and insensitive (snn2 genotype) to SnTox2 averaged 2.9 and 2.5 , respectively, indicating that the $T s n 1-$ SnToxA interaction played a relatively larger role in disease caused by Sn5 than did the Snn2-SnTox2 interaction. Lines with sensitivity to both effectors (Tsn1/Snn2 genotype) were significantly more susceptible than lines with sensitivity to only SnToxA (Tsn1/snn2 genotype) or only SnTox2 (tsn1/Snn2 genotype) (Table 1). Lines insensitive to both effectors (tsn1/snn2 genotype) were significantly more resistant than lines with sensitivity to only SnToxA (Tsn1/snn2 genotype) but they were not significantly different from those lines with sensitivity to only SnTox2 (tsn1/Snn2 genotype), indicating that a compatible Snn2-SnTox2 interaction played a significant role in disease susceptibility caused by $\mathrm{Sn} 5$ only in the presence of a compatible Tsn1-SnToxA interaction. Lines with sensitivity to only SnToxA (Tsn1/snn2 genotype) were significantly more susceptible than lines with sensitivity to only SnTox2 (tsn1/Snn2 genotype), further demonstrating that SnToxA plays a more significant role in disease caused by $\mathrm{Sn} 5$ than does SnTox2.

\section{Sequence analysis of the SnToxA gene in Sn4 and Sn5.}

We determined the DNA sequences of the SnToxA genes in isolates $\mathrm{Sn} 4, \mathrm{Sn} 5$, and $\mathrm{Sn} 6$, which is the isolate used by Friesen and associates (2007) (see Discussion) to determine the degree of SnToxA sequence variation among these isolates. Sequence data generated included the entire SnToxA coding region, $415 \mathrm{bp}$ of the $5^{\prime}$ flanking region, and $317 \mathrm{bp}$ of the $3^{\prime}$ flanking region, for a total of $1,319 \mathrm{bp}$. Comparative sequence analysis indicated that the SnToxA coding regions were $100 \%$ identical among the three isolates, and $\mathrm{Sn} 4$ and $\mathrm{Sn} 6$ shared identical $5^{\prime}$ and $3^{\prime}$ flanking regions (Fig. 3). Sn5 differed from $\mathrm{Sn} 4$ and Sn6 at two nucleotide positions in the $5^{\prime}$ region and five positions in the $3^{\prime}$ region. All seven polymorphisms were transition mutations, and none occurred within predicted regulatory elements, including the promoter.

\section{Sn4 and Sn5 SnToxA expression in planta.}

We used relative quantitative polymerase chain reaction (RQ-PCR) to evaluate Sn4 and Sn5 SnToxA expression in planta. Samples were collected from the susceptible cultivar Grandin and the resistant line BR34 at 6, 26, 48, and $72 \mathrm{~h}$ after inoculation with spores of isolates $\mathrm{Sn} 4$ or $\mathrm{Sn} 5$. For Sn4-inoculated plants, relative SnToxA expression was nearly identical for both Grandin and BR34, which both showed a significant increase at $26 \mathrm{~h}$ after inoculation followed by a steady decrease to near the 6-h levels at $72 \mathrm{~h}$ (Fig. 4).

Both wheat lines showed a pattern of expression for SnToxA produced by $\mathrm{Sn} 5$ similar to that observed for $\mathrm{Sn} 4$. However, the expression level of SnToxA produced by $\mathrm{Sn} 5$ at $26 \mathrm{~h}$ in Grandin was more than twice the relative level observed for Sn4 and, in BR34, the SnToxA expression level was approximately $1.4 \times$ the level observed in Sn4.

\section{DISCUSSION}

Most host-pathogen interaction research is focused on mechanisms of resistance, the host defense response, pathogen avirulence effectors, and ETI, which are all aspects of host interactions with bacteria or pathogens with biotrophic lifestyles. Less is known about host interactions with necrotrophic pathogens and mechanisms of susceptibility. The wheat-S. nodorum system relies largely on compatible interactions between necrotrophic effectors (HST) and corresponding host genes that mediate effector recognition. Until now, it was assumed that a compatible host gene-effector interaction in the wheat$S$. nodorum system would likely play a consistent and static role in conferring susceptibility, regardless of the host or pathogen backgrounds involved.

Infiltrations of the differential genotypes with culture filtrates of $S$. nodorum isolates $\mathrm{Sn} 4$ and $\mathrm{Sn} 5$ indicated that they both produce SnToxA and SnTox2. Therefore, the evaluation of disease caused by these two isolates in side-by-side experiments allowed us to study and compare the roles and effects of compatible Tsn1-SnToxA and Snn2-SnTox2 interactions. Together, the two interactions accounted for 50 and $60 \%$ of the variation in disease caused by $\mathrm{Sn} 4$ and $\mathrm{Sn} 5$, respectively. Although these two interactions are the predominate factors governing disease, other loci with relatively minor effects were also detected (data not shown) that accounted for additional variation, as was also observed in the BG population by Liu and associates (2006) and Friesen and associates (2007) for reaction to isolates Sn2000 and Sn6, respectively.

For the isolate $\mathrm{Sn} 4$, the effects of the Tsn1-SnToxA and Snn2-SnTox 2 interactions in causing disease were additive and nearly identical. In contrast, compatible Tsn1-SnToxA and Snn2-SnTox2 interactions did not contribute equally to disease caused by Sn5. There are several possible explanations for this phenomenon but, because all isolates were evaluated on the same host population, the differences most likely lie within the pathogen. Genetic variation within the coding sequences of the SnToxA and SnTox2 genes could exist among isolates, and certain variable codons important for affinity could cause some variants to be more efficient in binding to putative receptors or other targets leading to more rapid and extensive disease symptoms. However, sequence analysis of the SnToxA gene and flanking regions from isolates $\mathrm{Sn} 4, \mathrm{Sn} 5$, and $\mathrm{Sn} 6$ indicated that sequence polymorphisms are not likely to be the cause of the differences in susceptibility levels conferred by these three isolates. Alternatively, it is possible that the isolates differ in their degree of effector gene expression. Sn4 may express both effectors in approximately equal amounts, whereas Sn5 may express SnToxA at higher levels than SnTox2. Our results indicate that this scenario is the most likely because Sn5 expressed SnToxA at significantly higher levels (approximately twofold) than did Sn4. Given this result, it is quite possible that the SnTox2 gene is also differentially expressed among these isolates but the isolation of the SnTox2 gene is needed before appropriate experiments can be conducted to test this hypothesis.

It is interesting to note that expression of SnToxA in both isolates showed a marked increase in transcriptional accumulation $26 \mathrm{~h}$ after inoculation, followed by a rapid decline. Plants

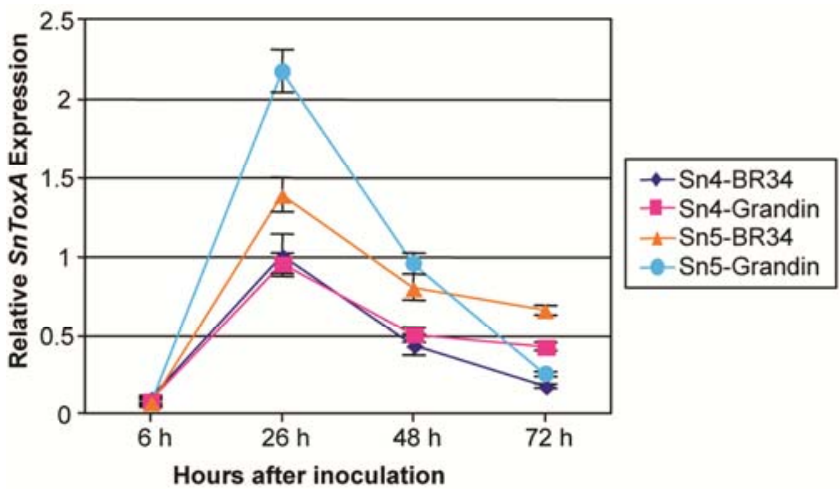

Fig. 4. Transcriptional expression of SnToxA in leaves of 'Grandin' and 'BR34' wheat at 6, 26, 48, and 72 h postinoculation with Stagonospora nodorum isolates $\mathrm{Sn} 4$ and $\mathrm{Sn} 5$. 
are placed in a mist chamber under high humidity conditions immediately after inoculation, and kept there for $24 \mathrm{~h}$ before being transferred to a growth chamber. Pathogen penetration begins several hours after inoculation and, therefore, occurs while in the mist chamber. The 26-h time point occurs after plants have been transferred to the growth chamber and have resided there for $2 \mathrm{~h}$. Therefore, this time point likely coincides with a reduction in growth on the leaf and attempted penetrations and an increase in intercellular growth and proliferation, at which time it would bode well for necrotrophic effectors to be produced and secreted. It appears that the expression of SnToxA is maximized at this time point, after which rapid cell death and eventual formation of lesions followed by asexual sporulation occur.

Typically, when multiple compatible interactions are operational in the wheat $-S$. nodorum system, the elimination of one compatible interaction by site-directed disruption of an effector gene (Friesen et al. 2006; Liu et al. 2009) leads to increased significance of the remaining compatible host gene-effector interactions. However, the average disease reaction across the population will decrease because the effects of compatible interactions are usually additive and, therefore, fewer compatible interactions leads to decreased levels of disease. In the case of $\mathrm{Sn} 4$ and $\mathrm{Sn} 5$, both isolates have the ability to produce the same effectors (i.e., SnToxA and SnTox2) but they are presumably expressed at different levels. This results in a different level of significance for each interaction but, interestingly, not a significant change in overall disease reaction severity across the population $(\mathrm{Sn} 4=2.8$; $\mathrm{Sn} 5=2.7)$ or in the susceptible parent Grandin ( $\mathrm{Sn} 4=4.7$; Sn5 = 4.8), which harbors both Tsn1 and Snn2. In the case of Sn5, the reduced severity caused by the Snn2-SnTox2 interaction, which we would speculate is due to reduced expression of SnTox2, is likely compensated for by increased expression of SnToxA, leading to an overall reaction type nearly identical to that caused by $\mathrm{Sn} 4$.

Friesen and associates (2007) evaluated the BG population for disease caused by the $S$. nodorum isolate Sn6, which also produces SnToxA and SnTox2. Although this was conducted in a separate experiment and caution is needed when making comparisons, it is interesting to note that, with Sn6, a compatible $S n n 2-S n T o x 2$ interaction played a more significant role in causing disease than did a Tsn1-SnToxA interaction (Friesen et al. 2007). In regression analysis, the Snn2-SnTox 2 and Tsn1-SnToxA interactions explained 47 and $20 \%$ of the variation, respectively. Each of the four genotypic classes were significantly different, with lines sensitive to both SnToxA and SnTox 2 being the most susceptible and lines insensitive to both effectors being the most resistant; however, lines with sensitivity to only SnTox2 (tsn1/Snn2 genotype) were significantly more susceptible than lines with sensitivity to only SnToxA (Tsn1/snn2 genotype). Therefore, all three SnToxAand SnTox2-producing isolates ( $\mathrm{Sn} 4, \mathrm{Sn} 5$, and $\mathrm{Sn} 6)$ differ in their reliance on SnToxA and SnTox 2 to cause disease (i.e., for $\mathrm{Sn} 4$, the two effectors contribute equally to disease; for Sn5, SnToxA contributes more to disease than SnTox2; and for Sn6, SnTox 2 contributes more to disease than SnToxA).

Other research involving the characterization of the Tsn1SnToxA interaction has also indicated that the degree of significance conferred by this interaction varies depending on the isolate used. Friesen and associates (2006) reported that SnToxA produced by two different S. nodorum isolates, Sn2000 and SN15, also had significantly different effects on disease development in the BG population. The Tsn1-SnToxA interaction in Sn2000-inoculated plants accounted for $64 \%$ of the variation whereas, in SN15-inoculated plants, it accounted for 34\%.

From this work, we cannot determine the reason for the differential expression of SnToxA in $\mathrm{Sn} 4$ and Sn5. Our analysis of the $5^{\prime}$ region of the $S n T o x A$ gene in $\mathrm{Sn} 5$ revealed only two nucleotides that differed from the same region of Sn4 SnToxA, and neither occurred within the predicted promoter region. It is possible that these, or the polymorphisms identified in the $3^{\prime}$ region, affect binding of regulatory factors that could alter transcription levels. However, Sn4 and Sn6 were identical within the SnToxA region evaluated. Although we did not evaluate expression of SnToxA in Sn6, the Tsn1-SnToxA interaction of Sn6-inoculated plants contributed more to disease (Friesen et al. 2007) than in Sn4-inoculated plants, suggesting that these two isolates also express SnToxA at different levels. This would suggest that factors influencing SnToxA expression differences among these isolates are not due to sequence-based differences within the 1,319 bp of the SnToxA locus analyzed in this work. Instead, expression differences may be due to differences in genes responsible for governing the regulation of SnToxA.

It is worth noting that another necrotrophic effector, Ptr ToxB, confers virulence in a dosage-dependent manner (Amaike et al. 2008). Ptr ToxB is produced by the wheat pathogen $P$. tritici-repentis and causes chlorosis in wheat lines that harbor the Tsc2 gene (Abeysekara et al. 2010; Friesen and Faris 2004; Orolaza et al. 1995). The Ptr ToxB gene is present in multiple copy numbers among virulent isolates (Martinez et al. 2004) and copy number appears to be correlated with the degree of virulence (Amaike et al. 2008; Ciuffetti et al. 2010), suggesting that, like SnToxA, increased expression of ToxB results in an increase in the level of disease severity. In addition, Strelkov and associates (2006) reported that the ToxB gene was expressed in both conidia and mycelia of a highly virulent isolate but only in conidia of less virulent and avirulent isolates, suggesting differences in regulatory mechanisms associated with virulence. Additional research is needed to determine the reasons for differential regulation of SnToxA, ToxB, and probably other effector genes, among relevant isolates.

The advantage of a necrotrophic pathogen harboring an arsenal of effectors in order to exploit an array of diverse host genotypes in a natural population is obvious. However, it may not be beneficial or necessary for a pathogen to produce all effectors at the same level at all times, because it would be rare that a single host genotype would harbor all the targets for which effectors are being produced. Other than SnToxA and SnTox2, Sn4 and Sn5 have been shown to produce many more effectors in culture (T. L. Friesen, unpublished data) and, therefore, some form of regulation of these effectors would be an evolutionary advantage for the pathogen. It is yet to be determined whether selection pressure applied by the population frequency of host effector recognition genes (e.g., Tsn1 and Snn2) may influence pathogen regulation of effector gene expression.

This research demonstrates that $S$. nodorum effectors can be differentially expressed in different isolates, and differential expression is directly related to disease significance. Future work will focus on identifying the mechanisms responsible for regulating effector gene expression, the cloning of additional $S$. nodorum effector genes so that expression of additional effectors can be monitored, and evaluating effector gene expression and their contributions to disease susceptibility in different isolates and genetic backgrounds to further understand the interactions associated with the wheat-S. nodorum pathosystem, which may serve as a useful model for other hostnecrotroph pathosystems.

\section{MATERIALS AND METHODS}

\section{Plant materials.}

The mapping population used to quantify the effects of compatible Tsn1-SnToxA and Snn2-SnTox2 interactions consisted 
of 118 RI lines derived from the BG population. Marker development and molecular mapping in the BG population was previously described (Liu et al. 2005; Zhang et al. 2009). The RI differential lines BG261 (sensitivity to only SnToxA) and BG223 (sensitivity to only SnTox2) were described by Friesen and associates (2006 and 2007, respectively). All plants were grown and maintained as described by Liu and associates (2004a).

Fungal cultures, culture filtrate production, and bioassays.

The $S$. nodorum isolate LDN03Sn4 ( $\mathrm{Sn} 4)$ was collected from a wheat field near Langdon, ND, U.S.A. in 2003 and isolate BBC03Sn5 (Sn5) was collected from a wheat field near Moorhead, MN, U.S.A. in 2003. Culture filtrates of $S$. nodorum were produced as previously described (Liu et al. 2004a). Infiltrations of the differential lines BG261 and BG223 and the parental lines BR34 and Grandin were done to verify that SnToxA and SnTox 2 were produced by $\mathrm{Sn} 4$ and $\mathrm{Sn} 5$. Briefly, approximately $25 \mu \mathrm{l}$ of active culture filtrate was infiltrated into a fully expanded secondary leaf using a 1-ml syringe without the needle. The boundaries of the infiltration site were marked with a felt pen. After infiltration, all plants were moved to a growth chamber at $21^{\circ} \mathrm{C}$ with a 12 -h photoperiod. Sensitive lines were characterized by necrosis within the infiltrated area whereas no reaction was visible in the infiltrated region of the insensitive lines (Fig. 1).

\section{Conidial inoculations}

with $S$. nodorum isolates $\mathrm{Sn} 4$ and $\mathrm{Sn} 5$.

For disease evaluation, individual lines of the BG population were inoculated with conidia of either $\mathrm{Sn} 4$ or $\mathrm{Sn} 5$. The parents and RI lines of the BG population were each grown in single cone-tainers (Stuewe and Sons, Inc., Corvallis, OR, U.S.A.), with three plants of each line per cone-tainer. Plants were placed in racks of 98 consisting of 60 lines and bordered with Grandin wheat to reduce any edge effects. Methods for producing inoculum and inoculating plants were done as described by Liu and associates (2004b). Plants were inoculated when the second leaf was fully expanded. Complete BG populations were inoculated with either $\mathrm{Sn} 4$ or $\mathrm{Sn} 5$ and populations of each inoculation were placed together in $100 \%$ relative humidity at $21^{\circ} \mathrm{C}$ for $24 \mathrm{~h}$, and then placed in the same controlled chamber under a $12-\mathrm{h}$ photoperiod at $21^{\circ} \mathrm{C}$. Disease ratings were done at 7 days postinoculation by scoring lesions on the second leaf using the 0 -to- 5 scale described by Liu and associates (2004b). Three completely randomized replicates were completed for the entire population, along with parental lines for each isolate.

\section{Statistical analysis.}

Mean separation for the genotypic means was determined by the use of Fisher's protected least significant difference at $\alpha$ $=0.05$. QTL analysis was conducted using the Map Manager QTX software package (Manly et al. 2001) as previously described (Friesen et al. 2007, 2008b; Liu et al. 2005, 2006) by performing composite interval regression analysis to determine the effects of compatible Tsn1-SnToxA and Snn2-SnTox2 interactions and the amount of phenotypic variation explained by the interactions. The genotypic data for $T s n 1$ and Snn2 were assembled into a multiple regression model to determine the proportion of the total variation in disease explained by the two effector sensitivity genes. The coefficient of determination $\left(R^{2}\right)$ value represents the amount of variation explained.

\section{SnToxA gene sequencing.}

Genomic DNA of Sn4 and Sn5 isolates was extracted using the Biosprint 15 DNA plant kit (Qiagen, Chatsworth, CA,
U.S.A.). Full-length ToxA genomic sequence was amplified using primers ToxA $\mathrm{Xbal}_{2}$.F (5'-AACTTGTATACTAGAGGAT CCGATGGAATCCATGG $-3^{\prime}$ ) and ToxA Xbal.R (5'-TTACC TATTCTAGAGGCGCCCGTGATAAATGTTT-3'). PCR was conducted using $1 \mu \mathrm{l}$ of Advantage cDNA Polymerase Mix (Clontech, Palo Alto, CA, U.S.A.), $100 \mathrm{ng}$ of DNA, $200 \mu \mathrm{M}$ dNTPs, $1 \times$ buffer, and $0.4 \mu \mathrm{M}$ forward and reverse primers in a $25-\mu l$ reaction. The PCR program started with $94^{\circ} \mathrm{C}$ for $4 \mathrm{~min}$; followed by 35 cycles of $94^{\circ} \mathrm{C}$ for $30 \mathrm{~s}, 60^{\circ} \mathrm{C}$ for $30 \mathrm{~s}$, and $72^{\circ} \mathrm{C}$ for $2 \mathrm{~min}$; and a 10 -min final elongation step. Products from three independent PCR reactions were separated on $2 \%$ agarose gel and gel purified using the QIAquick Gel Extraction Kit (Qiagen). Fragments were cloned using the TA Cloning Kit (Invitrogen, Carlsbad, CA, U.S.A.) and sequenced by the Iowa State University DNA Sequencing Facility using standard Sanger sequencing methods. Sequences were annotated according to Ciuffetti and associates (1997) and deposited in GenBank under accession numbers HM191251, HM191250, and HM191252 for the SnToxA locus from isolates Sn4, Sn5, and Sn6, respectively.

\section{Transcriptional expression analysis.}

Samples were collected from leaf tissue of BR34 (resistant) and Grandin (susceptible) wheat at 6, 26, 48, and $72 \mathrm{~h}$ after inoculation with either $\mathrm{Sn} 4$ or $\mathrm{Sn} 5$ and immediately frozen in liquid nitrogen. Total RNA was extracted from the leaf tissue samples using the RNeasy Plant Mini Kit (Qiagen). The firststrand cDNA was synthesized from $2 \mu \mathrm{g}$ of total RNA using the TaqMan reverse-transcription reagents (Applied Biosystems, Foster City, CA, U.S.A.). RQ-PCR performed on a 7500 Real-Time PCR System (Applied Biosystems) was used to quantify SnToxA gene expression in planta with the $S$. nodorum actin gene as an endogenous control using PCR primers ToxA.RT.F3 (5'-AACGCCAATACAGTGCGAGT-3') and ToxA.cod.1R (5'-GCTGCATTCTCCAATTTTCACG-3') for the SnToxA gene and Sn.actin.F (5'-CTGCTTTGAGATCC ACAT-3') and Sn.actin.R (5'-GTCACCACTTTCAACTCC-3') for the $S$. nodorum actin gene. Each experiment was repeated three times and all reactions were done in quadruplicate. The $20-\mu 1$ reaction contained $1 \times$ SYBR PCR Master Mix, $0.25 \mu \mathrm{M}$ each primer, and $5 \mu \mathrm{l}$ of twofold diluted cDNA. The thermocycler program used for SnToxA gene expression was as follows: $10 \mathrm{~min}$ of preincubation at $95^{\circ} \mathrm{C}$ followed by 40 cycles for $15 \mathrm{~s}$ at $95^{\circ} \mathrm{C}$ and $1 \mathrm{~min}$ at $57^{\circ} \mathrm{C}$. A dissociation program was performed after each RQ-PCR assay to confirm specific amplification.

Templates to determine the amplification efficiency of SnToxA and the $S$. nodorum actin gene consisted of five twofold dilutions of cDNA $(1: 2,1: 4,1: 8,1: 16$, and 1:32) in quadruplicate. Raw cycle threshold $\left(\mathrm{C}_{\mathrm{T}}\right)$ values were averaged for each dilution. $\mathrm{C}_{\mathrm{T}}$ numbers were plotted against logarithm cDNA input, and linear regression was used to draw the best-fit line. Amplification efficiency was calculated based on slope, where efficiency $=100 \% \times \tan ^{-1}\left(\operatorname{slope}^{-1}\right) / 45$. Amplification efficiencies were higher than $95 \%$ for both gene systems. Sample $C_{T}$ values were averaged after omitting outlying $\mathrm{C}_{\mathrm{T}}$ values for each gene. Sample averages were linearized using the $2^{-\Delta \Delta C T}$ method (Livak and Schmittgen 2001).

\section{ACKNOWLEDGMENTS}

This research was supported by United States Department of Agriculture (USDA) Agricultural Research Service CRIS project 5442-22000033-00D and by the National Research Initiative competitive grant number 2008-35301-19248 from the USDA National Institute of Food and Agriculture (NIFA) to J. D. Faris and by the Agricultural and Food Research Initiative (AFRI) grant number 2009-04265 from USDA NIFA to T. L. Friesen. 


\section{LITERATURE CITED}

Abeysekara, N. S., Friesen, T. L., Liu, Z. H., McClean, P. E., and Faris, J. D. 2010. Marker development and saturation mapping of the tan spot Ptr ToxB sensitivity locus $T s c 2$ in hexaploid wheat. Plant Genome 3:179-189.

Adhikari, T. B., Bai, J., Meinhardt, S. W., Gurung, S., Myrfield, M., Patel, J., Ali, S., Gudmestad, N. C., and Rasmussen, J. B. 2009. Tsn1-mediated host responses to ToxA from Pyrenophora tritici-repentis. Mol. Plant-Microbe Interact. 22:1056-1068.

Amaike, S., Ozga, J. A., Basu, U., and Strelkov, S. E. 2008. Quantification of $\operatorname{Tox} B$ gene expression and formation of appressoria by isolates of Pyrenophora tritici-repentis differing in pathogenicity. Plant Pathol. 57:623-633.

Ballance, G. M., Lamari, L., and Bernier, C. C. 1989. Purification of a host-selective necrosis toxin from Pyrenophora tritici-repentis. Physiol. Mol. Plant Pathol. 35:203-213.

Chu, C. G., Faris, J. D., Xu, S. S., and Friesen, T. L. 2010. Genetic analysis of disease susceptibility contributed by the compatible Tsn 1SnToxA and Snn1-SnTox1 interactions in the wheat-Stagonospora nodorum pathosystem. Theor. Appl. Genet. 120:1451-1459.

Ciuffetti, L. M., Tuori, R. P., and Gaventa, J. M. 1997. A single gene encodes a selective toxin causal to the development of $\tan$ spot of wheat. Plant Cell 9:135-144.

Ciuffetti, L. M., Manning, V. A., Pandelova, I., Betts, M. F., and Martinez, J. P. 2010. Host-selective toxins, Ptr ToxA and Ptr ToxB, as necrotrophic effectors in the Pyrenophora tritici-repentis-wheat interaction. New Phytol. 187:911-919.

Eyal, Z. 1981. Integrated control of Septoria diseases of wheat. Plant Dis. 65:763-768

Eyal, Z., Scharen, A. L., Prescott, J. M., and van Ginkel, M. 1987. The Septoria Diseases of Wheat: Concepts and Methods of Disease Management. CIMMYT, Mexico, D.F.

Faris, J. D., and Friesen, T. L. 2009. Reevaluation of a tetraploid wheat population indicates that the Tsn1-ToxA interaction is the only factor governing Stagonospora nodorum blotch susceptibility. Phytopathology 99:906-912.

Faris, J. D., Anderson, J. A., Francl, L. J., and Jordahl, J. G. 1996. Chromosomal location of a gene conditioning insensitivity in wheat to a necrosis-inducing culture filtrate from Pyrenophora tritici-repentis. Phytopathology 86:459-463.

Faris, J. D., Haen, K. M., and Gill, B. S. 2000. Saturation mapping of a generich recombination hot spot region in wheat. Genetics 154:823-835.

Faris, J. D., Zhang, Z., Lu, H. J., Lu, S., Reddy, L., Cloutier, S., Fellers, J. P., Meinhardt, S. W., Rasmussen, J. B., Xu, S. S., Oliver, R. P., Simons, K. J., and Friesen, T. L. 2010. A unique wheat disease resistance-like gene governs effector-triggered susceptibility to necrotrophic pathogens. Proc. Natl. Acad. Sci. U.S.A. 107:13544-13549.

Friesen, T. L., and Faris, J. D. 2004. Molecular mapping of resistance to Pyrenophora tritici-repentis race 5 and sensitivity to Ptr ToxB in wheat. Theor. Appl. Genet. 109:464-471.

Friesen, T. L., and Faris, J. D. 2010. Characterization of the wheat-Stagonospora nodorum disease system: What is the molecular basis of this quantitative necrotrophic disease interaction? Can. J. Plant Pathol. 32:20-28.

Friesen, T. L., Stukenbrock, E. H., Liu, Z. H., Meinhardt, S. W., Ling, H., Faris, J. D., Rasmussen, J. B., Solomon, P. S., McDonald, B. A., and Oliver, R. P. 2006. Emergence of a new disease as a result of interspecific virulence gene transfer. Nat. Genet. 38:953-956.

Friesen, T. L., Meinhardt, S. W., and Faris, J. D. 2007. The Stagonospora nodorum-wheat pathosystem involves multiple proteinaceous hostselective toxins and corresponding host sensitivity genes that interact in an inverse gene-for-gene manner. Plant J. 51:681-692.

Friesen, T. L., Faris, J. D., Solomon, P. S., and Oliver, R. P. 2008a. Hostspecific toxins: Effectors of necrotrophic pathogenicity. Cell Microbiol. 10:1421-1428.

Friesen, T. L., Zhang, Z., Solomon, P. S., Oliver, R. P., and Faris, J. D. 2008b. Genetic characterization of a novel wheat-Stagonospora nodorum host-selective toxin interaction and its role in disease suscepti- bility. Plant Physiol. 146:682-693.

Haen, K. M., Lu, H.-J., Friesen, T. L., and Faris, J. D. 2004. Genomic targeting and high-resolution mapping of the Tsn1 gene in wheat. Crop Sci. 44:951-962.

Hammond-Kosack, K. E., and Rudd, J. J. 2008. Plant resistance signaling hijacked by a necrotrophic fungal pathogen. Plant Signal. Behav. 3:993995.

King, J. E., Cook, R. J., and Melville, S. C. 1983. A review of the Septoria diseases of wheat and barley. Ann. Appl. Biol. 103:345-374.

Liu, Z. H., Faris, J. D., Meinhardt, S. W., Ali, S., Rasmussen, J. B., and Friesen, T. L. 2004a. Genetic and physical mapping of a gene conditioning sensitivity in wheat to a partially purified host-selective toxin produced by Stagonospora nodorum. Phytopathology 94:1056-1060.

Liu, Z. H., Friesen, T. L., Meinhardt, S. W., Ali, S., Rasmussen, J. B., and Faris, J. D. 2004b. Quantitative trait loci analysis and mapping of seedling resistance to Stagonospora nodorum leaf blotch in wheat. Phytopathology 94:1061-1067.

Liu, Z. H., Anderson, J. A., Hu, J., Friesen, T. L., Rasmussen, J. B., and Faris, J. D. 2005. A wheat intervarietal genetic linkage map based on microsatellite and target region amplified polymorphism markers and its utility for detecting quantitative trait loci. Theor. Appl. Genet. 111:792-794

Liu, Z. H., Friesen, T. L., Ling, H., Meinhardt, S. W., Oliver, R. P. Rasmussen, J. B., and Faris, J. D. 2006. The Tsn1-ToxA interaction in the wheat-Stagonospora nodorum pathosystem parallels that of the wheat-tan spot system. Genome 49:1265-1273.

Liu, Z. H., Faris, J. D., Oliver, R. P., Tan, K.-C., Solomon, P. S., McDonald, M. C., McDonald, B. A., Nunez, A., Lu, S., Rasmussen, J. B., and Friesen, T. L. 2009. SnTox3 acts in effector triggered susceptibility to induce disease on wheat carrying the Snn3 gene. PLoS Pathol. e1000581. Published online.

Livak, K. J., and Schmittgen, T. D. 2001. Analysis of relative gene expression data using real-time quantitative PCR and the 2(-delta delta C(T) method. Methods 25:402-408.

Lorang, J. M., Sweat, T. A., and Wolpert, T. J. 2007. Plant disease susceptibility conferred by a 'resistance' gene. Proc. Natl. Acad. Sci. U.S.A. 104:14861-14866.

Lu, H. J., Fellers, J. P., Friesen, T. L., Meinhardt, S. W., and Faris, J. D. 2006. Genomic analysis and marker development for the Tsn1 locus using bin-mapped ESTs and flanking BAC contigs. Theor. Appl. Genet. 112:1132-1142.

Manly, K. F., Cudmore, R. H. Jr., and Meer, J. M. 2001. Map Manager QTX, cross platform software for genetic mapping. Mamm. Genome 12:930-932.

Martinez, J. P., Oesch, N. W., and Ciuffetti, L. M. 2004. Characterization of the multiple copy host-selective toxin gene, ToxB, in pathogenic and nonpathogenic isolates of Pyrenophora tritici-repentis. Mol. Plant-Microbe Interact. 22:665-676.

Nagy, E. D., and Bennetzen, J. L. 2008. Pathogen corruption and site-directed recombination at a plant disease resistance gene cluster. Genome Res. 18:1918-1923

Orolaza, N. P., Lamari, L., and Ballance, G. M. 1995. Evidence of a hostspecific chlorosis toxin from Pyrenophora tritici-repentis, the causal agent of tan spot of wheat. Phytopathology 85:1282-1287.

Pandelova, I., Betts, M. F., Manning, V. A., Wilhelm, L. J., Mockler, T. C., and Cuiffetti, L. M. 2009. Analysis of transcriptome changes induced by Ptr ToxA in wheat provides insights into the mechanisms of plant susceptibility. Mol. Plant 2:1067-1083.

Strelkov, S. E., Kowatsch, R. F., Ballance, G. M., and Lamari, L. 2006. Characterization of the ToxB gene from North African and Canadian isolates of Pyrenophora tritici-repentis. Physiol. Mol. Plant Pathol. 67:164-170.

Wolpert, T. J., Dunkle, L. D., and Ciuffetti, L. M. 2002. Host-selective toxins and avirulence determinants: What's in a name? Annu. Rev. Phytopathol. 40:251-285.

Zhang, Z., Friesen, T. L., Simons, K. J., Xu, S. S., and Faris, J. D. 2009. Identification, development and validation of markers for markerassisted selection against the Stagonospora nodorum toxin sensitivity genes Tsn1 and Snn2 in wheat. Mol. Breed. 23:35-49. 